\title{
Una aproximación a las creencias populares de los romanos: las Lemurias, ¿respeto o temor?
}

\author{
An approach to the popular beliefs of the Romans: \\ the Lemuriae, respect or fear
}

\author{
Teresa Espinosa MartíneZ ${ }^{1}$
}

\begin{abstract}
RESUMEN
ABSTRACT

El temor a lo relacionado con los muertos hace que el hombre siempre se haya servido de todos los recursos a su alcance para protegerse de ellos. Un reflejo de esta idea aparece al efectuar un análisis sobre el rito que nos ocupa: las Lemuria, una antigua festividad romana que denota al mismo tiempo respeto y temor ante unas creencias ancestrales.

\section{PALABRAS CLAVE:}

Feriae, manes, larvae, lemures, lemurias, paterfamilias.

Fear of deads makes people have always used all the resources at its disposal to protect them. A reflection about this idea appears to make an analysis of the ritual in this particular case: the Lemuria, an ancient Roman festival that shows respect and at the same time fear for ancestral beliefs.

KEY WORDS:

Feriae, manes, larvae, lemures, lemurias, paterfamilias.

Un pueblo tan pragmático como el romano ¿creía en fantasmas? Esta pregunta, planteada de forma tan simple provoca en nosotros, en principio, contradicción e incluso cierta hilaridad ¿Es posible que este hombre antiguo tan práctico, tan apegado a las cosas terrenas como su casa, su alimento diario, su familia o su pueblo, pueda pensar en espectros, aparecidos y seres maléficos? Vamos a intentar acercarnos a la idea que él podía tener de la muerte para intentar dilucidar si era el respeto a la divinidad funeraria o el miedo que sentía a la muerte lo que le impulsaba a mantener su culto. Para ello examinaremos uno de los festivales más antiguos celebrados en Roma con relación a los difuntos: las Lemuria.
\end{abstract}

\footnotetext{
1 Teresa Espinosa Martínez, tespimar1 @ telefonica.net, UNED.
} 
Un buen punto de partida para iniciar este trabajo podría ser la obra de E.O. James $^{2}$ en la que haciendo un estudio comparativo de las religiones cita a $\mathrm{E}$. Ty${ }$ lor $^{3}$, quien trató de establecer la «creencia en seres espirituales» como «definición mínima de religión», sugiriendo que «los antiguos filósofos dieron probablemente el primer paso con la deducción obvia de que todo hombre posee dos cosas que le pertenecen: una vida y un fantasma». Este mismo autor nos remite también a $\mathrm{H}$. Spencer ${ }^{4}$, que utilizando la expresión "culto a los antepasados» en su más amplio sentido - hasta incluir en ella el culto a todos los muertos, los de la misma sangre y los extraños-, llega a la conclusión de que éste es la raíz de toda religión.

El culto romano a los difuntos, ya fuera público o privado, tuvo un doble propósito: que los muertos sobrevivieran en la memoria de sus parientes y amigos y tratar de asegurar un medio directo de atención para sus restos mortales en la tumba: comodidad, refresco y renovación permanente de vida para sus espíritus inmortales 5 . No obstante, P. Veyne ${ }^{6}$ ha estudiado la aparente incredulidad de la mayoría de los romanos en el más allá: «imaginaron la muerte como la nada, un sueño eterno». Entonces ¿por qué se pretende el mejor de los acomodos, qué se teme? «Los muertos ¿son poderosos o débiles, temibles o propicios? Todos estos ritos responden a sentimientos muy elementales: los muertos que vegetan pobremente bajo tierra aspiran a retornar al hogar e incluso a invadir el mundo terreno; hay que limitar sus movimientos fantasmales sin llegar a disgustarlos: serían capaces de llevarse consigo a los vivos ${ }^{7} \gg$.

El tránsito hacia el más allá requería la protección de los dioses o un complejo ritual de purificación que evitara al individuo una existencia angustiosa en el otro mundo. El hombre romano al abandonar esta vida entraba en la comunidad deificada de los manes, donde perdido entre las sombras de los antepasados, dependía de la piedad de sus descendientes para continuar su culto y para que se buscara su descanso en un lugar inviolable, locus religiosus ${ }^{8}$. Pero el mero hecho de la muerte no transformaba en «divinidad» al difunto, antes debía recibir los funerales apropiados y era preciso que se le tributara los iusta. La inhumación regular ponía fin a este proceso, pero en el caso que se produjese un olvido, una irregularidad, o que el difunto no fuera enterrado, éste se convertiría en un fantasma sin descanso hasta el día en que sus allegados o parientes le hicieran justicia9 ${ }^{9}$. Lo que más se temía era la privación de sepultura. En Roma el entierro de los muertos era un de-

2 JAMES, E.O. (1973): Introducción a la historia comparada de las religiones. Madrid, p. 38.

3 TYLOR, E. (1871): Primitive Culture, I, Londres, p. 81.

${ }^{4}$ SPENCER, H. (1885): Principles of Sociology, I, Londres, p. 421.

${ }^{5}$ TOYNBEE, J.M.C. (1996): Death and Burial in the Roman World. Londres, p. 61.

6 VEYNE, P. (1987): «El imperio romano» en Historia de la vida privada. Madrid, Vol. I, p. 216.

7 BAYET, J. (1984): La religión romana. Historia política y psicológica. Madrid, p. 84.

${ }^{8}$ Existe numerosa bibliografía referente al espacio reservado para los enterramientos, a su legislación y tratamiento vid:: DUCOS, M.(1995): «Le tombeau, locus religiosus» en La mort au quotidien dans le monde romain. París, pp. 135-144; ANDRÉ, J.M. (1990): «L'espace des morts et l'espace des vivants dans la Rome classique" en La vie et la mort dans l'antiquite. Actes du colloque de Dijon, pp. 1-15; DE VISSCHER, F. (1963): Le droit des tombeux romains. Milán.

9 SCHEID, J. (1984): «Contraria facere». AION 6, pp. 117-139; SCHEID, J. (1991): La religión de Roma. Madrid, pp. 46-47. 
ber sagrado. Negar sepultura a un cadáver era condenar el alma muerta a errar sin descanso, y, en consecuencia, crear un peligro real para los vivos. «En este caso, el difunto, en vez de entrar a formar parte de los manes que recibían culto en la tumba familiar durante las fiestas de los muertos, corría peligro de convertirse en una sombra atormentada, uno de aquellos espíritus maléficos -lemures-que el cabeza de familia expulsaba de su casa, conforme a un rito ancestral ${ }^{10}$ 》.

Se hacía un gran esfuerzo para asegurar que la ceremonia, el ritual y el entierro fueran de forma que el difunto pudiera tener un viaje fácil hacia el siguiente reino. El error más leve podía perjudicar o dificultar este camino. El entierro, al mismo tiempo que un penoso proceso, ofrecía la posibilidad a los allegados de honrar y despedir al difunto, sin embargo, cuando todo el ritual terminaba, el dolor por la pérdida de la persona querida no desaparecía, por ello existían días especialmente señalados para los muertos en los que se les permitía volver a la vida para recordar a los vivos que se debía visitar a los difuntos. Quizás esto hizo que surgieran las primeras supersticiones, que apareciera el temor y, por tanto, que se tomaran precauciones al pensar que estos espíritus pudieran ser potencialmente dañinos si no se les obedecía. Existió un miedo general en estos días que poco a poco se fue convirtiendo en un tiempo solemne para tratar con la pena y recordar a los seres queridos que se plasmó en la visita a las tumbas de los antepasados como un modo de mantener con ellos una relación de concordia, reforzada además con el ofrecimiento de regalos, flores y alimentos, cosas que formarían parte de su vida de no haber muerto.

¿Cómo regulaba el Estado las relaciones entre vivos y muertos? ¿Por qué tenían los romanos un festival para celebrar un tiempo de fantasmas? ¿Cuál era la actitud de los devotos ante su culto? ¿Sentían miedo ante estas divinidades de ultratumba, o, en cambio, era sólo respeto y consideración? La respuesta habitual es que los fantasmas debían ser propiciados por temor a que pudieran dañar la ciudad o a su gente y que el culto rendido a los muertos respondiera a un respeto natural, pero también a una precaución, a una defensa.

El calendario estatal fijaba celebraciones regulares (feriae $)^{11}$ que, pese a su diversidad de origen y de ritual, tenían como fin garantizar la «paz» entre los muertos y los vivos, la mayor parte de las veces con una intención expiatoria. Dos períodos del año estaban consagrados a los muertos: el primero, relacionado con los espíritus de los parientes más cercanos y celebrado públicamente del 13 al 21 de febrero, las Parentalia, hacía que la ciudad se entregara durante esta novena a unas fiestas totalmente carentes de alegría. Los días, 9, 11 y 13 de mayo, se celebraban las Lemuria, que evocaban, por su parte, un temor ancestral: los difuntos,

10 SCHILLING, R. (1973): «Religión romana» en Historia Religionum. Vol. I: Religiones del pasado. Madrid, p. 471.

${ }_{11}$ Las feriae se pueden clasificar en feriae privatae, observadas por las familias de forma individual y feriae publicae, con carácter público. Posteriormente éstas últimas se subdividieron en tres categorías: feriae stativae, fiestas cuya fecha permanecía fija en el calendario, conceptivae, asignadas por sacerdotes o magistrados y las feriae imperativae, festividades improvisadas con motivo de una victoria o en épocas de crisis. 
bajo el nombre de lemures, volvían a sus antiguas viviendas, y era necesario apaciguar estas sombras, a la vez que alejarlas lo más rápidamente posible. Tenían, por tanto, a diferencia de las anteriores, un carácter privado ya que eran celebradas por cada familia, dentro de cada hogar ${ }^{12}$, aunque no podemos descartar que en algún momento pudieran tener también un culto público y desconozcamos la naturaleza de los sacrificios que pudieron ser ofrecidos en ellas. Su origen arcaico parece claro en cuanto a que aparecen ya citadas en el Fasti Antiates Maiores ${ }^{13}$, datado entre el 84 y el 46 a.C., el calendario más antiguo de la república romana que ha sobrevivido. Estas fiestas estaban consignadas como feriae stativae en días fijos, no sucesivos e impares, y con la consideración de nefasti, lo que conllevaba que los templos permanecieran cerrados y estuviera prohibida la celebración de matrimonios. El influjo funesto de estos días se extendía a todo el mes de mayo, habiéndose comprobado que, al parecer, los matrimonios celebrados durante este mes no duraban mucho ${ }^{14}$. Roma fijaba así en sus ritos el temor primitivo hacia los muertos y aparecidos y garantizaba el respeto debido a las tumbas.

En cuanto al origen de la palabra Lemuria aún no se ha explicado claramente pero se relaciona con Remo, de quien se dice que, una vez asesinado por su hermano Rómulo, se aparecía de noche para atormentar a Acca Larentia y a Faustulus $^{15}$. Ovidio nos dice que la sombra de Remo (llamada Remores), tras su muerte, suplicó un festival en su honor y que Rómulo concedió la petición estableciendo las Remuria. Con el tiempo la «r» se transformó en «l» lo que derivaría en las palabras lemures y Lemuria $^{16}$. Otra explicación del término Remuria estaría referida al lugar físico donde Remo fue ritualmente sacrificado y enterrado, asociándolo así al Aventino y a la fundación de Roma, y donde se celebrarían los primeros festivales en su honor. Posteriormente el día se relacionaría con todos las personas que fueron asesinadas o muertas prematuramente y eventualmente los nuevos significados que se le iban dando a este término terminaron por incluir a todos los muertos.

Las Remuria son una historia de fantasmas, un relato más poético que fidedigno; mientras hoy un historiador sería considerado poco ortodoxo por incorporar a su obra una tradición así, semejante casi a un cuento, antiguamente podría considerarse normal aunque, debe destacarse que ningún otro autor antiguo habla o menciona la aparición de Remo o cualquier otro rito dedicado a él, de lo que parece concluirse o bien que era una historia suficientemente conocida o que quizás Ovidio arregló la historia y la escribió de forma más sencilla para llegar así a un mayor número de personas.

De los actos que tenían lugar durante las Lemurias todo lo que tenemos es el detallado relato que Ovidio hace del ritual que el paterfamilias celebraba en priva-

12 OVIDIO: Fastos, V, 419-429.

13 SCULLARD, H.H. (1981): Festivals and ceremonies of the Roman Republic. Londres, p. 47.

14 Fastos, V, 485-490.

15 Faustulus fue el pastor que descubrió a los dos hermanos, y Acca Larentia, su mujer, quien los amantó con su leche: Fastos, IV, 854; V, 455; HORACIO: Epodas, II, 2, 208.

16 Fastos, V, 479-484. Debe destacarse que esta definición, para varios estudiosos modernos, es insostenible tanto por razones lingüïsticas como históricas. 
do en la medianoche del último día y con el que se daban por concluidas las celebraciones. El oferente se levantaba a medianoche, cuando había callado todos los perros y dormían toda clase de aves, yendo con los pies descalzos, y haciendo chasquear los dedos, para que en su silencio no salieran al encuentro ninguna sombra ligera. Se lavaba tres veces las manos con agua pura de una fontana, se volvía y tomaba en su boca habas negras, que luego tiraba detrás de sí diciendo: "iYo tiro estas habasi ¡Por ellas me salvo yo y los míosj». Repetía esta expresión nueve veces seguidas, sin volver la vista atrás, porque pensaba que la sombra le seguía y las recogía. De nuevo tocaba el agua, tañía un objeto de bronce y rogaba a la sombra que saliera de su casa, increpándole también por nueve veces «Manes de mis padres, salid». Se volvía, por fin, entendiendo que había realizado el ceremonial con pureza ${ }^{17}$.

La narración de Ovidio es precisa e informativa y nos presenta en un primer momento al cabeza de familia como oficiante. Es él quien tenía la obligación de realizar los cultos familiares y como uno más de sus deberes debí propiciar y ahuyentar a los espíritus de la casa para proteger a los suyos, en un momento preciso, (nox ubi iam media est somnoque silentia praebet, et canis et variae conticuistis aves) y de una forma determinada, descalzo, sin atadura alguna en sus pies (habent gemini vincula nulla pedes). Recurría, además, a un gesto exacto para que nada le pudiera perturbar (signaque dat digitis medio cum pollice iunctis, occurrat tacito ne levis umbra sibi). Su intención era alimentar a los espectros con el lanzamiento de habas (umbra putatur colligere (fabas) et nullo terga vidente sequi) que completa el rito central. Los fantasmas están alimentados, queda ahuyentarles y esto se realiza de tres formas: lavándose las manos nuevamente (rursus aquam tangit), haciendo ruido con implementos de bronce (Temesaeaque concrepat aera) y repitiendo nueve veces la fórmula de despedida (manes exite paterni).

El temor a lo relacionado con los muertos, con los espíritus malignos, hace que el hombre siempre se haya servido de todos los recursos a su alcance para protegerse de ellos. Un reflejo de esta idea aparece al efectuar un análisis sobre el rito que nos ocupa ya que hallamos en él rasgos arcaicos, mágicos, ctónicos, supersticiosos y religiosos que se entrelazan de modo que no nos permite delimitar con claridad si pertenecen a uno $u$ otro ámbito de creencias pero que tienen en común este único objetivo de defensa.

El ritual posee un claro carácter ctónico al estar relacionado con divinidades ultraterrenas y con los elementos que las identifican: la noche, el agua de fuente o el trato directo con los muertos, en este caso, ordenándoles; también el número nueve y las habas tienen una significación fúnebre en el mundo antiguo. Los elementos mágicos también aparecen constantemente en el relato: el vestido del celebrante, los gestos, las habas y la repetición de términos, son algunos de ellos. Es importante, sin embargo, recordar que el agua y el bronce también encajan en la categoría de elementos mágicos y que muchos de ellos pueden tener además

\footnotetext{
17 Fastos V, 429-445.
} 
una naturaleza religiosa ${ }^{18}$. Es precisamente la mención específica del bronce temeseo ${ }^{19}$ la que aporta al relato una forma arcaica.

En último lugar debemos afrontar el debate sobre la terminología ya que existe mas de un término para designar a los fantasmas en lengua latina y Ovidio utiliza al menos cuatro: umbrae, imago, manes y lemures. La idea común es que cada término denota una diferencia de calidad o de categoría de los muertos, pero la confusión se mantiene porque son utilizados indistintamente a pesar de su posible diferenciación. ¿Cuándo el pater familias realiza el rito, exorciza a sus abuelos, a fantasmas rebeldes o a los espíritus del inoportuno muerto? Intentaremos descubrir qué son los lemures examinando someramente la terminología y la interpretación encontrada tanto en fuentes antiguas como en estudios modernos.

Algunos autores diferencian entre lemures y manes dando a este último término un carácter arcaico al ser usado siempre en plural -recordemos que la idea más antigua que se tenía en Roma de los muertos era la de una pluralidad bastante indiferenciada, aunque designara el alma de un solo difunto, que se ha explicado por las costumbres funerarias asimiladas de la cultura Terramara-, en género masculino y haciéndolo derivar de la palabra manis con el significado de «los buenos, los ilustres ${ }^{20}$. H. Rose ${ }^{21}$ matiza que no hay ninguna palabra conocida, de fecha anterior a Augusto, que definiera inequívocamente el fantasma de una persona muerta o su espíritu, y que la fórmula dis manibus aparece precisamente en ese tiempo.

Otra de las ideas recurrentes a la hora de analizar esta cuestión es la de la confusión por parte de Ovidio al dirigirse a los lemures como manes y la interpretación de la fórmula Manes exite paterni. A este respecto los estudiosos se dividen: W. Fowler ${ }^{22}$ considera la fómula como una apelación eufemística de los espíritus hostiles por el pater familias, y en esta misma línea incide $\mathrm{G}$. Wissowa ${ }^{23}$ al sugerir que el término lemures podría tratarse de una licencia poética utilizada para designar a los fantasmas en las fuentes antiguas. J. Frazer ${ }^{24}$, al contrario, acepta la fórmula como acertada y sostiene que Ovidio cree en los lemures como los antepasados muertos. Por su parte, K. Latte ${ }^{25}$, parece estar de acuerdo con los que dis-

18 Los ritos y elementos mágico-religiosos en la Antigüedad han sido objeto de numerosos estudios. Podrían citarse entre otros: HIDALGO, M.J.: «La magia y la religión en las obras de Apuleyo» Zephyrus, XXX-XXXI, pp. 223-230; TUPET, A.M. (1985): «Rites magiques dans l'Antiquité romaine». ANWR 16.3, pp. 2590-2675; MILLETT, M. (2000): Burial, society and context in the Roman world. Oxford; MIRECKI, P.(2002): Magic and ritual in the ancient world. Leiden; OGDEN, D. (2002): Magic, witcraft, and ghosts in the Greek and Roman words, Oxford.

19 Témesa era una antigua ciudad de los Abruzzos, donde existían minas de cobre que se utilizaban para la acuñación de moneda.

20 Entre ellos, DUMEZIL, G. (1970): Archaic Roman Religión, Chicago, pp. 365-367, siendo ésta la idea más extendida. 135.

21 ROSE, H. (1930): «Ancient Italian Beliefs Concerning the Soul», Classical Quarterly, 24, pp. 129-

22 FOWLER, W. (1969): The Roman Festivals. New York, pp. 106-109.

23 WISSOWA, G. (1893-1978): «Lemuria». Paulys Real Encyclopädie der Classischen Altertumswissenschaft, 34 vols., Stuttgart, Vol 12.2, pp. 1931-1933.

24 FRAZER, J. (1951): Ovid: Fasti. Londres, p. 424.

25 LATTE, K. (1960): Römische Religionsgeschichte. Munich, p. 99. 
tinguen lemures de di parentes y piensa que Ovidio los confundió al usar la fórmula, y que obviamente plantea un problema de interpretación al mezclar conceptos diferentes: espíritus buenos y fantasmas desfavorables. En cuanto al carácter de estos, W. Fowler destaca que mientras las Lemuria eran celebradas en dias nefasti, otro festival romano para los muertos, las Feralia, incluido dentro de las Parentalia, sucedían en días fasti, de lo que concluye que los muertos de las Lemuria son expresamente de naturaleza hostil, y que regresan a sus antiguas casas con el fin de buscar un entierro digno.

Todos estos estudios nos obligan necesariamente a revisar, de forma sucinta, las fuentes antiguas para intentar contestar a los muchos interrogantes que se plantean, aunque no siempre vamos a hallar respuestas concretas, ya que las referencias son escasas y a veces no muy aclarativas. Si bien existen pruebas, tanto en la literatura romana como en la epigrafía, que sugieren que la denominación ordinaria para los muertos en época tardo-republicana y a principios del Imperio fue Manes o Dii Manes, también se utilizarían frecuentemente otros términos como umbra, imago, animus, anima, simulacrum o effigies. Pero, encontramos otras dos expresiones que designan a fantasmas, específicamente a fantasmas nocivos: lemures y larvas, y que a diferencia de lo dicho anteriormente, no aparecen ni en epitafios ni en poesía de la época. Este hecho pudo deberse, según $G$. Thaniel ${ }^{26}$, al aspecto tabú que estas palabras pudieron tener y que las hizo inadecuadas para la poesía, en tanto que manes podría ser una palabra eufemística, aceptable y utilizada con frecuencia.

Una de las pocas citas que se conservan de Mecenas tiene relación con los lemures: «a la busca de comida y bebida aparecen en nuestras moradas y pasan su muerte a la espera» en referencia a la necesidad que tienen los espíritus de continuo sustento. Otro de los primeros ejemplos lo encontramos en Horacio «nocturnos lemures portentaque Thessala rides» ${ }^{27}$. El poeta no explica qué son los lemures simplemente los adjetiva como nocturnos, asociándolos por tanto a las cosas que inspiran miedo. Esta misma cualidad la encontramos también en Ovidio: «nocturna Lemuria, sacri» 28 . Otro autor, Persio, en una línea muy similar apunta uno de sus rasgos, negros «tum nigri lemures ovaque rupto ${ }^{29} »$. Así, las únicas cosas que se pueden concluir de estos autores consisten en que los lemures pertenecen al reino de las supersticiones, que se temieron y que eran negros, no necesariamente malos, sino más bien asociados con poderes ctónicos. En sí mismos quizás no fueran malignos, pero tenían la connotación de estar unidos a cosas impuras y espantosas, por tanto se etiquetan como mal.

Porfirio comentando el pasaje de Horacio, dice que los lemures que vagaban durante la noche habían muerto prematuramente y eran temibles (umbras va-

26 THANIEL, G. (1973) : «Lemures and Larvae». The American Journal of Philology. Vol. 94, $\mathrm{n}^{\circ} 2$, pp. 182-187.

27 Epodos, II, 2, 209.

28 Fastos, V, 421.

${ }^{29}$ Sátiras V, 185. 
gantes hominum ante diem mortuorum et ideo metuendas). Distintos autores coinciden en señalar que cuando la muerte ocurre de forma violenta o prematura, el tipo de espíritu es esencialmente distinto, o al menos habita en distinto sitio. Virgilio, por ejemplo, sitúa en el limbo a aquellos que han muerto de forma injusta ${ }^{30}$, a los suicidas ${ }^{31}$, a las víctimas del $a m o r^{32}$ y a los caídos en la guerra ${ }^{33}$.

Otra referencia a los lemures procede de Varrón que dice que son lo mismo que las larvas y que a ellas les son lanzadas las habas durante las Lemuria «quibus temporibus en sacris fabam iactant ac dicunt se lemurios domo extra ianuam eicere» ${ }^{34}$. Paulo también sostiene esta idea (nam et Lemuralibus (faba) iacitur larvis) ${ }^{35}$ y Nonio Marcelo define a los lemures llamándoles larvas (larvas nocturnae et terrificationes imaginum et bestiarum ${ }^{36}$. Estas tres citas que nos sitúan, dentro del ritual que narra Ovidio, en el momento en que se lanzan las habas para exorcizar a los fantasmas, causa un nuevo problema al introducir una clase diferente de espíritus, las larvas. A diferencia de lemures y manes, larvas como vocablo, incluye formas de adjetivo, participio y verbo ${ }^{37}$, pero más allá de la terminología ¿podemos distinguir entre lemures y larvas?.

Tanto Ovidio como Persio indican que lemures y manes son los mismos, no haciendo ninguna mención a las larvas. Es Apuleyo quien diferencia los tres términos: «El espíritu del hombre, después de haber salido del cuerpo, se convierte en una especie de demonio que los antiguos llamaban lemures. Aquellos difuntos que eran buenos y cuidaban de sus descendientes se llamaban lares familiares, pero los que eran inquietos, turbulentos y nocivos, los que asustaban a los hombres con apariciones nocturnas, se llamaban larvae y cuando no era seguro en qué se había convertido el alma del difunto, si en lar o en larva, se le llamaba mane „38. Esta confusión llega hasta San Agustín, que citando a Plotino, escribe: «Dice también que las almas de los hombres se tornan Lares si son de buen mérito; si de malo, Lemures o Larvas, y dioses Manes, si es incierto si son buenos o malos sus merecimientos» 39 .

30 Eneida VI, 430-433.

31 Idem, VI, 434-439.

32 Idem VI, 440 ss.

33 Idem VI, 477-493.

34 Frase 18 de Vita Populi Romani.

35 PAULUS: Digesto XI.7.44.

36 NONIUS MARCELUS: De compendiosa doctrina. Leipzig, 1903.

37 Larvalis, parecido a un espectro, a un espíritu malo, de muerte; larvatus, poseído por malos espíritus, demente y larvare, poseer con malos espíritus.

38 De Deo Socr. 15: «Est et secundo significatus speces daemonum animus humanus emeritis stipendiis vital corporesuo abiurans. Hunc vetere Latina lengua reperio Lemurem dictitatum. Ex hisce ergo Lemuribus qui posterorum suorum curam sortitus placato et quieto numine domum possidet, Lar dicitur familiares; qui vero ob adversa vital merita nullis (bonis) sedibus incerta vagatione ceu quodam exilio punitur, inane terriculamentum bonis hominibus, ceterum malis noxium, id genus plerique Larvas perhibent. Cum vero incertum est, quae cuique forum sortitio evenerit, utrum Lar sit an Larva, nomine Manem deum nuncupan: scilicet et honoris gratia dei vocabulum additum est...».

39 Ciu. Dei, 9, 11: «Dicit quidem et animas hominum daemons esse et ex hominibus fieri lares, si boni meriti sunt; lemures, si mali, seu laruas; manes autem deos dici, si incertum est bonorum eos seu malorum esse meritorum...». 
Larva es el término para el esqueleto de plata que en la cena de Trimalción un esclavo pone sobre la mesa ${ }^{40}$. Séneca no deja lugar a dudas sobre las larvas afirmando que son esqueletos: "larvarum habitum nudis ossibus cohaerentium» "41. Plauto usa el término para referirse a los malos espíritus que infectan a los hombres como enfermedad mental y como una criatura no deseada e inoportuna «Laruae hunc atque intemperiae insaniaeque agitant senem ${ }^{42}$.

Así, con cada autor sucesivo, surge una nueva clase de espíritu o un entendimiento diferente de ellos. Si se asume que al principio los muertos eran para los romanos una masa indeterminada el llegar a explicaciones más complicadas quizás se deba a la influencia de la cultura griega.

¿Pero por qué regresan los muertos? ¿Qué buscan? D. Felton ${ }^{43}$ perfila tres motivos primarios que los difuntos tienen para volver: la venganza, el consuelo y el entierro. La idea del regreso para vengar su muerte aparece en varios ejemplos: la madre de Nerón, después de que éste la hubiera asesinado, lo atormentó hasta tal punto que convocó un mago que le exorcizase ${ }^{44}$; o como Galba no dejaría a su sucesor, Otón, atormentándole durante toda la noche ${ }^{45}$. La vuelta para advertir o aconsejar a los vivos se refleja también en la obra de Apuleyo: el fantasma de un padre vuelve para advertir a su hija que su madrastra habría cometido un crimen ${ }^{46}$; pero la razón más popular que los fantasmas tienen para volver en reclamar un entierro apropiado. Ya hemos mencionado el hecho de que la persona que era abandonada insepulta, que no era enterrada, no podía descansar después de la muerte. Esta creencia nos ayuda a comprender el significado de la terrible maldición que lanza Eneas sobre el cuerpo de un orgulloso enemigo muerto: «... No vendrá a depositarte en la tierra una madre amorosa; no dejará tu cuerpo bajo el peso de la tumba en tu patria. Serás abandonado a las aves de presa o arrojado a los abismos del mar donde vendrán los peces voraces a lamer tus heridas... »47. Calígula frecuentó los jardines de Lamia hasta que sus hermanas lo enterraron ${ }^{48}$. Plauto nos cuenta en Mostellaria que Diapontio fue asesinado por su anfitrión y enterrado bajo la casa que él ahora atormenta ${ }^{49}$. Plinio nos describe como una casa es visitada porque el cuerpo de alguien fue ocultado debajo de la casa. Una vez que los huesos fueron descubiertos y correctamente enterrados el tormento cesó $^{50}$.

40 Petronio, 34, 8.
41 Epigramas, 34.

42 Aulularia, 642.

43 FELTON, D. (1999): Haunted Greece and Rome. Austin, University of Texas, pp. 8-12.

${ }_{44}$ SUETONIO, Nerón, 34, ante lectum iacens per ominia piaculorum genera Manes Galbae, a quo deturbari expellique se viderat, propitiare remptasse.

${ }^{45}$ SUETONIO, Otón, 7. Es interesante destacar que el autor usa el término manes para designar al fantasma de la madre de Nerón y de Galba, pero utiliza umbra para referirse al fantasma de Calígula que pasea por los jardines de Lamia.

${ }_{46}$ APULEYO, Metamorfosis, 9, 31.

47 VIRGILIO, Eneida, 10, 557-560.

48 SUETONIO, Calígula, 59.

49 PLAUTO: Mostellaria, 497-500.

50 PLINIO: Hist. Nat., VII, 27, 5-11. 
El culto a los muertos es uno de los actos fundamentales que diferencia a los hombres del resto de los seres vivos. Todas las culturas tienen modos diferentes de tratar a los muertos. Actuar de forma que parezca que los muertos permanecen todavía vivos de algún modo, adornando cementerios, ofreciendo regalos y libaciones, rezando y participando en entierros y estableciendo fiestas realizadas en su memoria, parece denotar la idea de que la muerte no es el final. Como hemos visto Roma antigua no fue ninguna excepción. Los romanos honraron a sus muertos con grandes monumentos en cementerios y a lo largo de caminos; con sencillos funerales y con grandes cortejos fúnebres. Es posible que estas fiestas tuvieran componentes que no fueron tanto para honrar a los muertos como para protegerse de ellos. Religión y culto a los muertos se dan la mano con el fin de garantizar un descanso pacífico en un espacio del que a veces se duda, pero con el que no se pueden correr riesgos.

\section{FUENTES}

AGUSTIN (San).

\section{Civitas Dei.}

APULEYO = Lucius Apuleius.

Apología.

Metamorfosis.

De Deo Socratis.

CICERÓN = Marcus Tullius Cicero.

De Legibus.

FESTO $=$ Sextus Pompeius Festus

De verborum significatu, cum Pauli Diacono

epitoma (ed. W.M. Lindsay. Leipzig, 1913).

HORACIO = Quintus Horatius Flaccus.

Epístolas.

Satiras.

Epodos.

LIVIO = Titus Livius.

Ab urbe condita.

LUCRECIO = Titus Lucretius Carus.

De Rerum natura.

MACROBIO = Aurelius Macrobius.

Saturnalia.

NONIO MARCELO = Nonius Marcellus.

De compendiosa doctrina (ed. W.M. Lindsay. Leipzig, 1903). 
OVIDIO = Publius Ovidius Naso .

Fasti.

PERSIO = Persius Flaccus.

Saturae.

PLAUTO = Titus Maccius Plautus.

Mostellaria.

PLINIO = Gaius Plinius Secundus.

Naturalis Historiae.

PORFIRIO.

PROPERCIO = Sextus Propertius.

Carmina.

SÉNECA = Lucius Annaeus Séneca.

Apocolocintosis.

SERVIO = Servius

Ad Aeneidem, Commentarii in Vergilium ad Aeneidem.

SUETONIO = Gaius Suetonius Tranquilus, .

De vita Caesarum.

TÁCITO = Gaius Cornelius Tacitus .

Annales Historiae.

TERTULIANO = Q. Septimius Tertulianus.

De anima.

VIRGILIO = Publius Vergilius Mar.

Aeneidem.

\section{BIBLIOGRAFÍA}

ANDRÉ, J.M. (1990): «L'espace des morts et l'espace des vivants dans la Rome classique» en La vie et la mort dans l'antiquite. Actes du colloque de Dijon, pp. 1-15.

BANTI, S. (1929): «ll culto dei morti nella Roma antichissima». SIFC 7, pp. 171-198.

BAYET, J. (1971): Croyances et rites dans la Rome antique. Paris.

BAYET, J. (1984): La religión romana. Historia política y psicológica. Madrid.

BELOT, E.( 1992): «Les dieux et les morts dans l'Antiquité». Archéologia, 277, pp. 52-59.

BOYANCÉ, P.(1972): «Le symbolique funéraire des Romains». Études sur la religion romaine. Roma, pp. 299-307.

BURRISS, E.E. (1972): Taboo, magic, spirits. A study of primitive elements in roman religion. Greenwood Press, Westport, Connecticut.

CUMONT, F. (1942): Recherches sur le symolisme funéraire des romains. Paris.

DAREMBERG, C. \& SAGLIO, E. (1877-1919): Dictionnaire des antiquités grecques et romaines. 10 vols. Paris.

DE VISSCHER, F. (1963): Le droit des tombeux romains. Milán.

DELATTE, L. (1936): «Recherches sur quelques fêtes moviles du calendrier romain». L'Antiquité classique V, pp. 381-404. 
DESCHAMPS, L.(1995): «Rites funéraires de la Rome républicaine» en La mort au quotidien dans le monde romain. París, pp. 171-180.

DUCOS, M. (1995): «Le tombeau, locus religiosus» en La mort au quotidien dans le monde romain. París, pp.135-144.

DUMEZIL, G. (1970): Archaic Roman Religion, Chicago.

DUPONT, F. (1987): «Les morts et la memoire: la masque fúnebre». La mort, les morts, Caen, pp. 167-172.

FELTON, D. (1999): Haunted Greece and Rome. Austin, University of Texas.

FOWLER, W. (1969): The Roman Festivals. New York.

FRAZER, J. (1951): Ovid: Fasti. Londres.

GRAF, F. (1994): La magie dans l'antiquite greco-romaine, Paris.

GUILLÉN, J. (1980): Vrbs Roma. Vida y costumbre de los romanos. Vol. III, Religión y ejército. Salamanca.

HIDALGO, M.J. (1980): «La magia y la religión en las obras de Apuleyo» Zephyrus, XXXXXXI, pp. 223-230.

HILD, J.A. (1877-1919): «Larvae» en Dictionnaire des antiquités grecques et romaines, Daremberg-Saglio, Vol. II-3, pp. 950 -953.

HILD, J.A. (1877-1919): «Lemures» en Dictionnaire des antiquités grecques et romaines, Daremberg-Saglio, Vol. II-3, pp. 1100-1101.

HINARD, F.(ed.) (1987): La mort, les morts et l'au de là dans le monde romain, Caen.

HINARD, F.(ed.) (1993): «La mort au quotidien dans le monde romain». Actes du colloque organisé para l'Université de Paris IV, Paris.

JACOBSEN, J.P.(1924): Les Manes. París.

JOBBÉ DUVAL, E.(1924): Les morts malfaisans: «Larvae, Lemures», d'après le droit et les croyances populaires des romains. París.

LATTE, K. (1960): Römische Religionsgeschichte. Munich.

LUX, J. (2004): Examination of the Lemures and the Lemuria, University of Florida.

MICHELS, A.K. (1967): The calendar of the Roman Republic. Princeton University Press.

MILLETT, M. (2000): Burial, society and context in the Roman world. Oxford.

MIRECKI, P. (2002): Magic and ritual in the ancient world. Leiden.

OGDEN, D. (2002): Magic, witcraft, and ghosts in the Greek and Roman words, Oxford.

ROSE, H.J. (1923): «Nocturnal funerals in Rome», Classical Quarterly, vol. 17, n 3-4, pp. 191-194.

ROSE, H.J. (1930): «Ancient Italian Beliefs Concerning the Soul», Classical Quarterly, 24, pp. 129-135.

ROSE, H.J.(1941): «Manes exiti paterni». Universidad California 12/6, pp. 89-94.

ROSE, H.J.(1948): «Keres and Lemuries», Harvard Theological Review 41, pp. 217 ss.

ROSE, H.J.(1948): Ancient Roman Religion. Londres.

SCHEID, J. (1984): «Contraria facere: reversements et déplacements dans les rites funéraires». Annali del Istituto Orientale di Napoli (AION), 6, pp. 117-139.

SCHEID, J. (1985): Religión et piété à Rome. Paris.

SCHEID, J. (1991): La religión de Roma. Madrid.

SCHILLING, R. (1973): «Religión romana» en Historia Religionum, Vol. I, Religiones del pasado. Madrid, pp. 435-482.

SCHILLING, R. (1979): Rites, cultes, dieux de Rome. Paris.

SCULLARD, H.H.(1981): Festivals and Ceremonies of the Roman Republic. Londres.

SPENCER, H. (1885): Principles of Sociology, I, Londres.

THANIEL, G. (1973) : «Lemures and Larvae». The American Journal of Philology. Vol. 94, n 2, pp. $182-187$.

TOUTAIN, J. (1966): Les cultes paiëns dans l'Empire romaine. Roma.

TOYNBEE, J.M.C. (1996): Death and Burial in the Roman World. Londres.

TURCAN, R. (1998): Rome et ses dieux. Pais.

TUPET, A.M. (1985): «Rites magiques dans l'Antiquité romaine». ANWR 16.3, pp. 2590-2675. 
TYLOR, E. (1871): Primitive Culture, I, Londres.

VACCAI, G. (1986): Le festi di Roma Antica. Roma.

VEYNE, P. (1987): «El imperio romano» en Historia de la vida privada. Madrid, Vol. I, pp. 21227.

WARDE FOWLER, W. (1899): The Roman Festivals of the period of the Republic. Londres. WISSOWA, G. (1971): Religion und kultus der Römer. Munich.

WISSOWA, G. (1893-1978): «Lemuria». Paulys Real-Encyclopädie der Classischen Altertumswissenschaft, 34 vols., Stuttgart, Vol 12.2, pp. 1931-1933.

WOLSKI, W. \& BERCIN I. (1973): «Contribution au problème des tombes romaines á dispositif pour les libations funéraires». Latomus, 32, pp. 370-379.

VV.AA. (1985): Religión, superstición y magia en el mundo romano. Encuentros en la Antigüedad, 1983, Cádiz. Universidad de Cádiz. 